\title{
High-throughput sequencing analyses of oral microbial diversity in healthy people and patients with dental caries and periodontal disease
}

\author{
TINGTAO CHEN $^{1 *}$, YAN SHI $^{2 *}$, XIAOLEI WANG ${ }^{1}$, XIN WANG $^{1}$, \\ FANJING MENG $^{1}$, SHAOGUO YANG ${ }^{1}$, JIAN YANG ${ }^{2}$ and HONGBO XIN ${ }^{1}$ \\ ${ }^{1}$ Institute of Translational Medicine, Nanchang University, Nanchang, Jiangxi 330031; \\ ${ }^{2}$ Department of Conservative Dentistry and Endodontics, Stomatological Hospital \\ of Nanchang University, Nanchang, Jiangxi 330006, P.R. China
}

Received April 2, 2016; Accepted March 7, 2017

DOI: $10.3892 / \mathrm{mmr} .2017 .6593$

\begin{abstract}
Recurrence of oral diseases caused by antibiotics has brought about an urgent requirement to explore the oral microbial diversity in the human oral cavity. In the present study, the high-throughput sequencing method was adopted to compare the microbial diversity of healthy people and oral patients and sequence analysis was performed by UPARSE software package. The Venn results indicated that a mean of 315 operational taxonomic units (OTUs) was obtained, and $73,64,53,19$ and 18 common OTUs belonging to Firmicutes, Bacteroidetes, Proteobacteria, Actinobacteria and Fusobacteria, respectively, were identified in healthy people. Moreover, the reduction of Firmicutes and the increase of Proteobacteria in the children group, and the increase of Firmicutes and the reduction of Proteobacteria in the youth and adult groups, indicated that the age bracket and oral disease had largely influenced the tooth development and microbial development in the oral cavity. In addition, the traditional 'pathogenic bacteria' of Firmicutes, Proteobacteria and Bacteroidetes (accounted for $>95 \%$ of the total sequencing number in each group) indicated that the 'harmful' bacteria may exert beneficial effects on oral health. Therefore, the data will provide certain clues for curing some oral diseases by the
\end{abstract}

Correspondence to: Professor Hongbo Xin, Institute of Translational Medicine, Nanchang University, 1299 Xuefu Road, Honggu, Nanchang, Jiangxi 330031, P.R. China

E-mail: hongboxin@yahoo.com

Dr Jian Yang, Department of Conservative Dentistry and Endodontics, Stomatological Hospital of Nanchang University, 49 Fuzhou Road, Nanchang, Jiangxi 330006, P.R. China

E-mail: jianyang399@hotmail.com.cn

${ }^{*}$ Contributed equally

Key words: high-throughput sequencing analyses, dental caries, periodontal disease, microbial diversity strategy of adjusting the disturbed microbial compositions in oral disease to healthy level.

\section{Introduction}

The oral cavity contains a large number of sites, which are suitable for the growth and assemblage of various microorganisms (1). To date, 700 phylotypes have been identified in the oral cavity and half of these microbes exist in any individual during their lifetime (2). These microorganisms, which are predominantly composed ofbacteria, may prevent oral colonization from exogenous organisms, indicating that they are indispensableto health (3). In addition, many normal oral inhabitants are associated with some oral diseases including dental caries, gingivitis, periodontitis, candidiasis, endodontic infections, orthodontic infections and peri-implantitis $(2,4-7)$. Therefore, it is crucial to investigate the actual microbe compositions inhealthy peopleand dental patients for understanding the relationship between bacteria and various oral diseases.

In general, bacterial infections within the oral cavity are polymicrobial in nature, and it is quite unusual for any oral disease caused by a single species (8). However, medical microbiologists have relied on culture techniques to elucidate the complexity of infections for decades, and these culture methods can only be used for identifying the 'culturable' bacteria that are growing relatively quickly and easily in laboratory media $(9,10)$, and it is technically difficult to separate and identify $>3-6$ species because of their various requirements of nutrition, $\mathrm{pH}$, temperature andoxygen, and the interspecies competition anddifferent concentrations of bacteria on the culture plate also increase the difficulties of screening $(11,12)$. To determine the roles of bacteria in oral diseases, it is necessary to define the full panoply of organisms within the human mouth, and high-throughput sequencing methods may be a useful method for alleviating this problem since it can detect almost all the DNA signatures of microorganisms within a specific environment, even those present inlow numbers or in a dormant metabolic state (13-15). 
As is already known, dental caries is a destructive condition of the dental hard tissues that can progress to inflammation and death of vital pulp tissue, with eventual spread of infection to the periapical area of the tooth and beyond (16). Conversely, periodontal diseases can involve in both the soft and hard tissues and are the most common inflammatory destructive conditions that affect humans (8). To eliminate the overestimation of the importance of species that are easily cultured and the underestimation of the fastidious organisms that may be highly prevalent and important in dental caries and periodontal diseases, high-throughput sequencing analyses were used in the present study.

\section{Materials and methods}

Ethical statement. The study was approved by the Ethical Committee of the Stomatological Hospital of Nanchang University and all participants provided written informed consent.

Patient selections and sampling. The patients only with typical dental caries and periodontal diseases were selected from the Stomatological Hospital of Nanchang University (Nanchang, China). Patients with dental caries were recorded if either cumulative caries (dmft/DMFT) or initial caries score was $>0$ or the Streptococcus mucans count was $\geq 105 \mathrm{CFU} / \mathrm{ml}$ (17), and periodontal disease was defined as two or more teeth with clinical attachment loss (CAL) or $\geq 4 \mathrm{~mm}$ (18). Then, the patients were divided into seven groups: Children-Control group (C.C, n=5, 6-18 years old), Children-Dental caries group (C.DC, $\mathrm{n}=8,6-18$ years old); Youth-Control group (Y.C, $\mathrm{n}=8$, 18-35 years old), Youth-Dental caries group (Y.DC, $n=8$, 18-35 years old); Adult-Control group (A.C, $n=7,35-60$ years old), Adult-Dental caries group (A.DC, $n=6,35-60$ years old), and Adult-Periodontitis group (A.P, $n=8,35-60$ years old). All of the subjects were Chinese, and those patients who were pregnant, lactating or had other systemic conditions were excluded. In addition, none of the subjects had received systemic antibiotics or periodontal therapy in the previous 6 months (19).

Stimulated whole saliva and oropharyngeal samples were collected over a 6-month period from healthy and dental patients. Subjects chewed a $1 \mathrm{~g}$ piece of paraffin wax for $1 \mathrm{~min}$, and after swallowing once, they expectorated secreted saliva into a sterile plastic $50 \mathrm{ml}$ tube several timesand this was kept frozen at $-20^{\circ} \mathrm{C}$ until processing. The oropharyngeal samples were collected using dry cotton to gently swab the posterior wall of the oropharynx in which the samples were directly suspended in a microtube containing $200 \mu \mathrm{l}$ lysis buffer [Tris $20 \mathrm{mM}$, EDTA $2 \mathrm{mM}$ (pH 8), Tween-20 1\%, proteinase K (Thermo Fisher Scientific, Inc., Waltham, MA, USA) $400 \mu \mathrm{g} / \mathrm{ml}$. Then, saliva samples with the same volume of $2 \mathrm{X}$ lysis buffer and the oropharyngeal lysates were mixed in a 1:10 ratio $(19,20)$, and $200 \mu \mathrm{l}$ mixture of saliva and oropharyngeal lysates from every patient in same group were mixed together for DNA extraction (21).

Extraction of genome DNA and high-throughput sequencing. Genomic DNA of each sample was extracted by a TIANamp Genomic DNA kit (Tiangen Biotech Co., Ltd., Beijing, China) combined with bead beating, as previously published (22). The extracted genomicDNA was used as the template to amplify the V3region of 16S rRNA genesusing primer of 515F/806R with the barcode. PCR reactions, pyrosequencing of the PCR amplicons and quality control of raw data were performed as described previously (23).

Bioinformatics and multivariate statistics. Paired-end reads from the original DNA fragments weremerged using FLASH (Fast Length Adjustment of Short Reads to Improve Genome Assemblies; http://www.cbcb.umd.edu/software/flash) to merge paired-end reads when at least some of the reads overlap the read generated from the oppositeend of the same DNA fragment, and paired-end reads were assigned to each sample according to the unique barcodes.

Then, sequences analysis was performed by UPARSE software package version 7.0.1001 (http://drive5.com/uparse/) using the UPARSE-operational taxonomic unit (OTU) and UPARSE-OTUref algorithms. In-house Perl scripts were used to analyze alpha (within samples) and beta (among samples) diversity. Sequences with $\geq 97 \%$ similarity were assigned to the same OTUs. Sequence was picked as a representative for each OTU, and the Ribosomal Database Project (RDP; http://rdp.cme.msu.edu/classifier/classifier.jsp) classifier was used to annotate taxonomic information for each representative sequence. Cluster analysis was preceded by unweighted UniFrac distance using the Quantitative Insights into Microbial Ecology software package version 1.8.0 (QIIME; http://qiime. org/index.html).

\section{Results}

Shared genera inhealthy peopleand dental patients. To compare the microbes in healthypeople and dental patients, 16S rRNA amplicon sequencing analysis was used to sequence the V4 hypervariable region, and the sequencing data was filtered to get the valid data. All the effective tags of all samples were clustered and those sequences with over $97 \%$ similarity were considered as one OTU. In total, $1,321,995$ usable raw sequences $(8,686$ unique sequences) and 2,207 OTUs were obtained from all the samples with an average of 315.3 OTUs per group (Table I). The Chaol and Shannon indexes were almost saturated and the rarefaction curve of every sample was able to enter the plateau phase (data not shown).

The Venn figure may reflect the difference between all groups. As presented in Fig. 1, therewere305 (C.C), 285 (C.DC), 305 (Y.C), 335 (Y.DC), 288 (A.C), 331 (A.DC), and 358 (A.P) OTUs in each group, and there were 237 common OUTs in the C.C, Y.C and A.C groups (healthy group, Fig. 1D); of which 73, 64, 53, 19 and 18 OUTs belonged to Firmicutes, Bacteroidetes, Proteobacteria, Actinobacteria and Fusobacteria respectively (data not shown). The common OUTs in the C.C, Y.C and A.C groups (disease group, Fig. 1E) was 248 , of which $79,64,54,19$ and 14 OUTs belonged to Firmicutes, Bacteroidetes, Proteobacteria, Fusobacteria and Actinobacteria respectively (data not shown). It should be noted that there were only threecommon OUTs in the C.DC, A.DC and Y.DC groups belonging to Spirochaetes (1 OTU) and Proteobacteria (2 OTUs), and the unique OUTs in the 
Table I. Number of total tags, taxon tags, unclassified tags, unique tags and OTUs from the oral cavity of healthy people and patients with dental caries and periodontal disease by high-throughput sequencing.

\begin{tabular}{lrcccc}
\hline Group & Total tags & Taxon tags & Unclassified tags & Unique tags & OTUs \\
\hline C.C & 182,851 & 181,759 & 0 & 1,092 & 305 \\
C.DC & 159,863 & 158,641 & 0 & 1,222 & 285 \\
Y.C & 206,886 & 205,408 & 0 & 1,478 & 305 \\
Y.DC & 151,942 & 151,027 & 0 & 915 & 335 \\
A.C & 188,805 & 187,700 & 0 & 1,105 & 288 \\
A.DC & 168,826 & 167,602 & 0 & 1,224 & 331 \\
A.P & 262,822 & 261,172 & 0 & 1,650 & 358 \\
Mean & 188,856 & $187,615.6$ & 0 & 1,241 & 315 \\
Total & $1,321,995$ & $1,313,309$ & 0 & 8,686 & 2,207 \\
\hline
\end{tabular}

OTUs, operational taxonomic units.
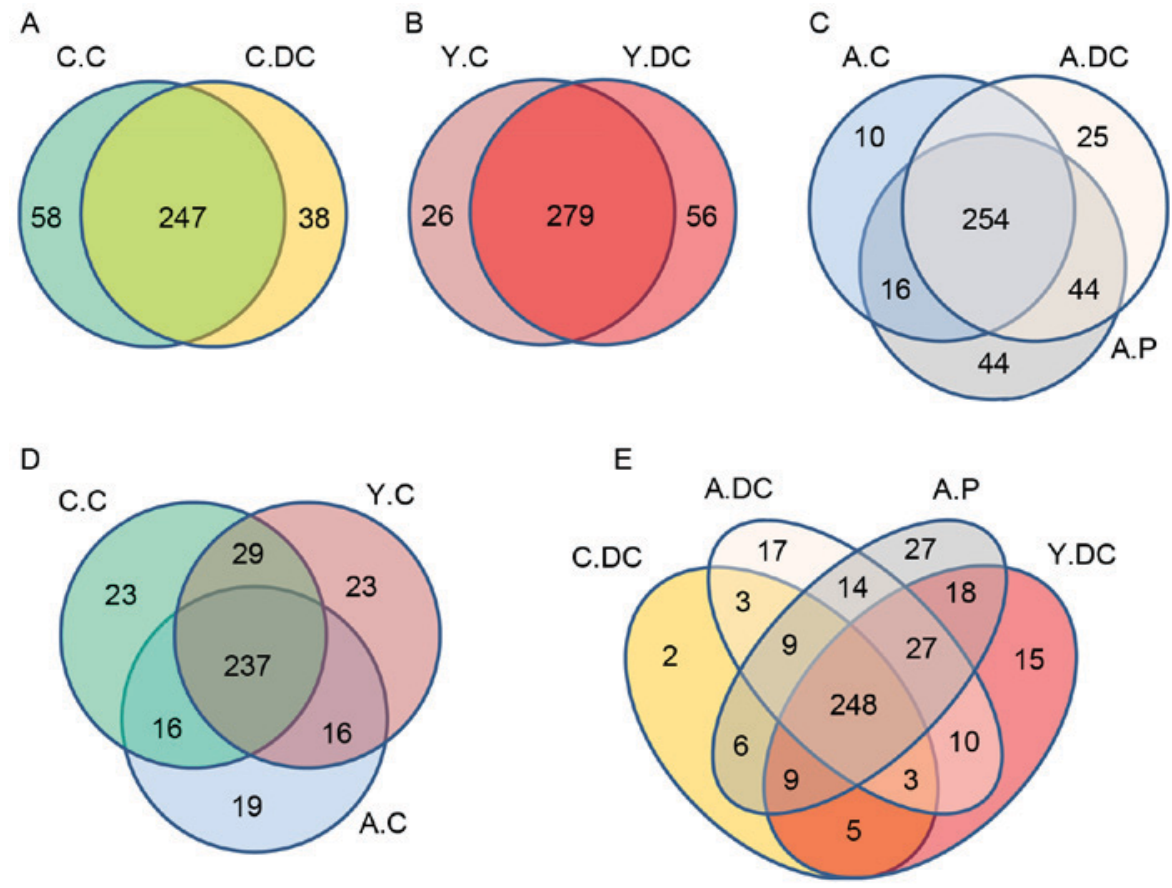

Figure 1. Scalar-Venn representation of the oral microbiota among C.C, C.DC, Y.C, Y.DC, A.C, A.DC and A.P groups. The shared OTUs between (A) C.C and C.DC; (B) Y.C and Y.DC; (C) A.C, A.DC and A.P; (D) C.C, Y.C and A.C; (E) C.DC, A.DC, A.P and Y.DC groups. C.C, Children-Control group; C.DC, Children-Dental caries group; Y.C, Youth-Control group; Y.DC, Youth-Dental caries group; A.C. Adult-Control group; A.DC, Adult-Dental caries group; A.P, Adult-Periodontitis group.

A.P group was 27, of which Actinobacteria only occupied 1 OUT, while Proteobacteria and Spirochaetesoccupied 5 and 3 OUTs, respectively.

Compositions and relative abundance of bacterial communities in phylum level. Based on the weighted UniFrac distance in phylum level, data for the top 10 microorganism populations were analyzed using unweighted Pair-group Method with Arithmetic Mean (UPGMA) to check the similarity between different groups (24). As presented in Fig. 2, Firmicutes, Proteobacteria and Bacteroidetes constituted the three commonest dominant phyla and accounted for $>95 \%$ of the total sequencing number in all groups, of which the percentage of Firmicutes was $75 \%$ in the Children-Control group (C-C, $\mathrm{n}=5,6-18$ years old), $48 \%$ in Youth-Control group (Y-C, $\mathrm{N}=8$, 18 35 years old) and 36\% in the Adult-Control group (A-C, $\mathrm{n}=7,35-60$ years old). The percentage for Proteobacteria was $10 \%$ in the C.C group, $32 \%$ in the Y.C group and $50 \%$ in the A.C group (Fig. 2), respectively. Notably, the percentage of Bacteroidetes was relatively stable, maintaining a ratio of $10 \%$ of the total sequencing number.

Compared with the healthy people, the percentages of Firmicutes and Proteobacteria were reduced from 75 to $45 \%$ in dental caries patients, and from 10 to $39 \%$ in the children group (C.C vs. C.DC), whereas the percentage of Firmicutes was increased from 48 to $58 \%$ and Proteobacteria was reduced 
A

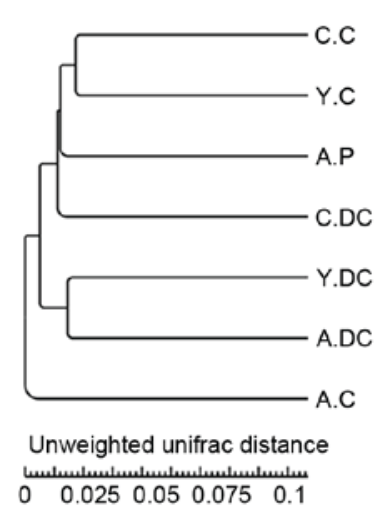

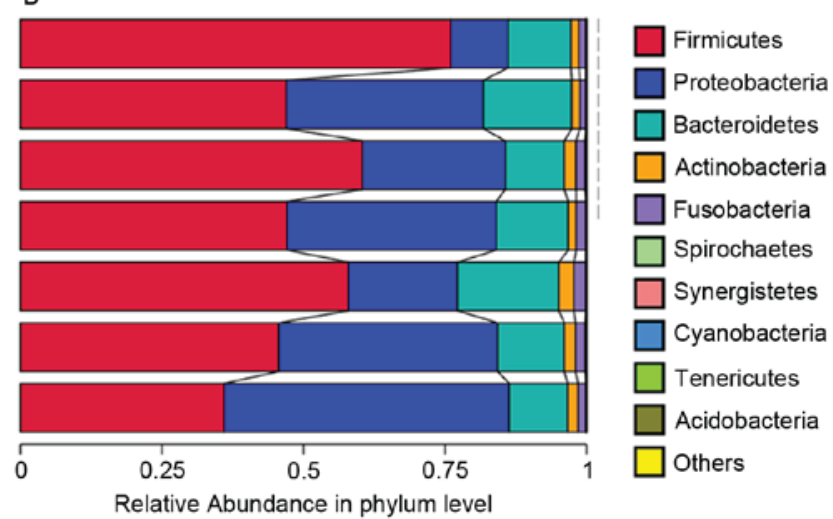

Figure 2. Composition and relative abundance of bacterial communities based 16S rDNA sequences in C.C, C.DC, Y.C, Y.DC, A.C, A.DC and A.P groups. (A) The UPGMA clustering tree Based on the Weighted UniFrac distance in phylum level. (B) The composition and relative abundance of bacterial communities in Kingdom, Phylum, Class, Family, Genus and Species levels. C.C, Children-Control group; C.DC, Children-Dental caries group; Y.C, Youth-Control group; Y.DC, Youth-Dental caries group; A.C. Adult-Control group; A.DC, Adult-Dental caries group; A.P, Adult-Periodontitis group.

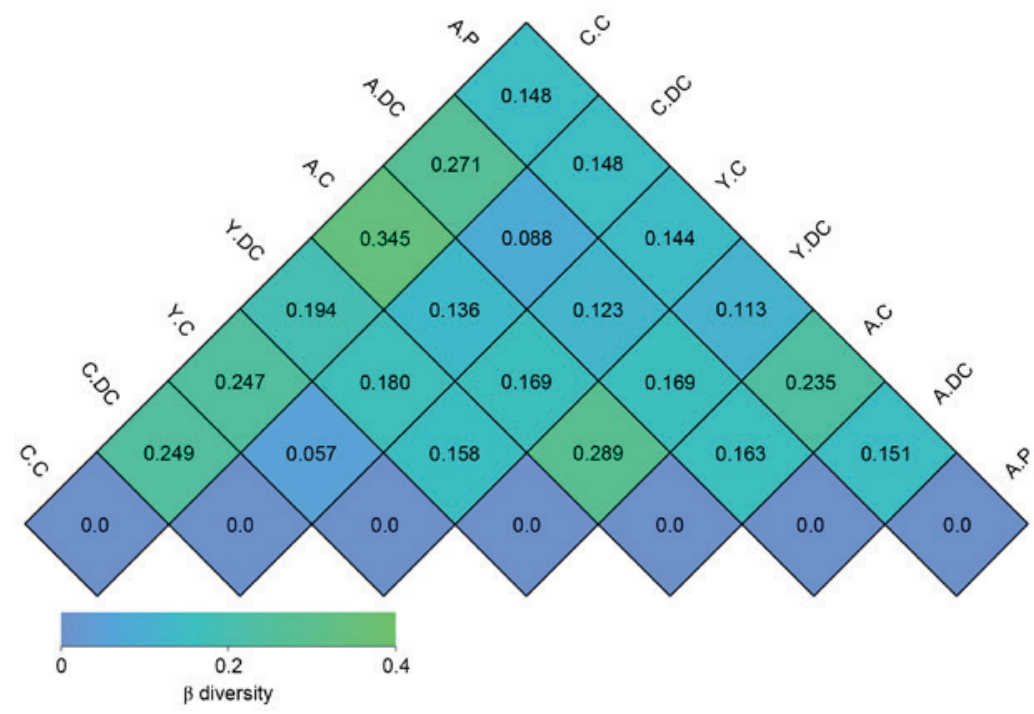

Figure 3. The heatmap of beta diversity index among C.C, C.DC, Y.C, Y.DC, A.C, A.DC and A.P groups. C.C, Children-Control group; C.DC, Children-Dental caries group; Y.C, Youth-Control group; Y.DC, Youth-Dental caries group; A.C. Adult-Control group; A.DC, Adult-Dental caries group; A.P, Adult-Periodontitis group.

from 32 to $19 \%$ in the youth group (Y.C vs. Y.DC). In the adult group, the dental caries made a slight increase in Firmicutes and a minor reduction in Proteobacteria, while the percentage of Firmicutes in the periodontitis group was greatly increased from 36 to $60 \%$, and Proteobacteria was reduced from 50 to $25 \%$.

As indicatedin Fig. 3, similarities of 0.247 (C.C vs. Y.C), 0.345 (C.C vs. A.C) and 0.136 (Y.C vs. A.C) were obtained in the healthy group, and 0.180 (C.DC vs. Y.DC), 0.088 (C.DC vs. A. DC) and 0.169 (Y.DC vs. A.DC) were obtained in the dental caries group.

Compositions and relative abundance of bacterial communities in genus level. To further investigate the relative abundance in groups C.C, C.DC, Y.C, Y.DC, A.C, A.DC and A.P, the top 10 genera were clustered. As demonstrated in Fig. 4, the dominant bacterial genera in all groups were Streptococcus and Neisseria. When dental caries occurred,

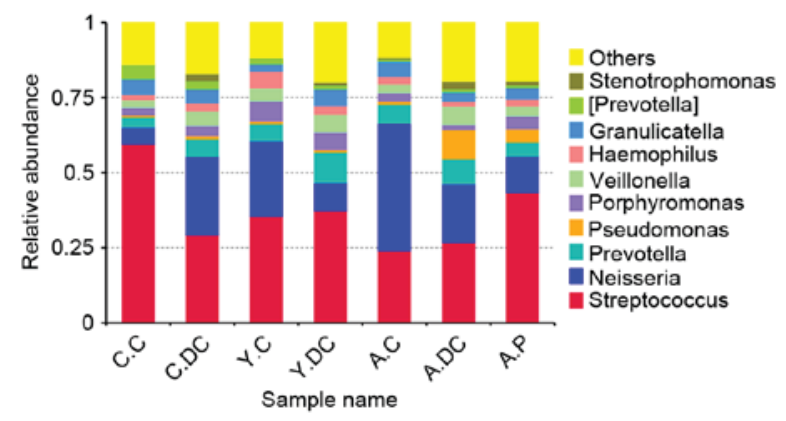

Figure 4. The relative abundance of the bacteria among C.C, C.DC, Y.C, Y.DC, A.C, A.DC and A.P groups at the genus level. C.C, Children-Control group; C.DC, Children-Dental caries group; Y.C, Youth-Control group; Y.DC, Youth-Dental caries group; A.C. Adult-Control group; A.DC, Adult-Dental caries group; A.P, Adult-Periodontitis group.

the relative abundance of Streptococcus reduced from 0.60 to 0.30 and Neisseria increased from 0.06 to 0.26 in the children 
group. Notably, the dental caries posed a slight effect on the relative abundance of Streptococcus, while Neisseria received a dramatic reduction in the youth group (from 0.26 to 0.09 ) and the adult group (from 0.42 to 0.19 ).

\section{Discussion}

Oral diseases appear to be the outcome of multiple microorganisms, and the complexity of the microbial community in the oral cavity has hampered the identification of a single etiological agent for dental caries (25). Although it has been demonstrated that Streptococcus sobrinus and Streptococcus mutans are acidogenic and serve an important role in caries initiation, the molecular techniques revealed that a high proportion of samples from cavities do not contain Mutans streptococci, whereas other acid-producing bacteria, e.g. Lactobacillus, Actinomyces or Bifidobacterium, existed (26,27). Therefore, it is important to investigate the actual microbial diversity in oral disease as a cooperation of microorganisms, rather than as individual organisms or species in the human mouth (28).

In previous studies, high-throughput sequencing had been used to investigate the microbiota in human and canine oral cavities $(5,19)$, which may ignore the importance of the age bracket on the tooth development and oral diseases. Moreover, the authors suggested that the low sample size in their studies may overlook inter-animal variations, as well as temporal changes that possibly occur, and that larger studies were required (5). To avoid the biodiversity loss during the DNA extraction process (the small size of each sample will make a huge loss of DNA) and to low the temporal changes of the oral microbiota, all the samples in the same age bracket were mixed and the mean number of 188,856.4 usable sequences (6-9 folds greater than the single sample in previous studies) and the saturated Chaol index and Shannon index (Table I) ensured their reliability for the future analysis.

In Fig. 1, the Venn figure demonstrated that a mean of 315 OTUs were obtained in each group, and 73, 64, 53, 19 and 18 common OUTs belonging to Firmicutes, Bacteroidetes, Proteobacteria, Actinobacteria and Fusobacteria, respectively, were identified in healthy people. As is already known, Firmicutes can survive in extreme conditions and made up the largest portion of the gut flora involved in energy resorption and obesity (29), Bacteroides are related to the metabolism of fat by absorbing nutrition and producing short-chain fatty acids (22), and Proteobacteria area major group of gram-negative bacteria (which includes a wide variety of pathogens) responsible for nitrogen fixation (30). Most of bacteria in these three phyla are regarded as pathogenic microorganisms, therefore the traditional treatment strategy to eradicate all the microorganisms in oral cavities using antibiotic drugs may not be suitable, as the 'pathogens' composed primarily of oral microorganisms and may serve a key role in oral health.

Based on the weighted UniFrac distance in phylum level, Firmicutes, Proteobacteria and Bacteroidetes accounted for $>95 \%$ of the total sequencing number in each group, and their total occupancy received little change between healthy and disease conditions (Fig. 2). However, the reduction of Firmicutesfrom 75 to $45 \%$ and the increase of Proteobacteria from 10 to $39 \%$ indicated the dental caries in the children group characterized by the raising of Firmicutes. Conversely, the dental caries made an increase of Firmicutes and a reduction of Proteobacteria in youth and adult groups, indicating that the age bracket was crucial for tooth development and microbial development. Considering the immature tooth development in the children group (6-18 years old) and the physiological depression in the adult group (35-60 years old), the microbial community in the youth group may be the most reasonable microbiota. Moreover, the high dissimilarity of 0.345 (C.C vs. A.C) in healthy patients and the high similarity of 0.088 (C.DC vs. A.DC) in diseased patients also confirmed the instability of the children and adult groups, when compared with the youth group (Fig. 3).

To further investigate the relative abundance among groups, the top 10 genera were clustered in Fig. 4, and Streptococcus and Neisseria were identified as the dominant bacteria genus in all groups. As with Fig. 2, the children group was characterized with the increase of Neisseria, while the youth group (from 0.26 to 0.09 ) and the adult group (from 0.42 to 0.19 ) were characterized by the reduction in Neisseria. The well-distributed bacteria were beneficial for the host and defended against invading pathogens (31), thus the more even microbial distribution in the Y.C group also confirmed their beneficial potential for pathogenic invasion.

In the present study, the microbial community of the human oral cavity had been explored using high-throughput sequencing, and these results indicated that the oral microbial communities were mainly composed of traditional 'pathogenic bacteria', and their ratio had been largely influenced by human age and presence of oral diseases. Therefore, it is necessary to further investigate the actual microbial diversity in healthy people and oral disease patients on a larger scale and identify the bacteria at species level; the unique bacteria should be screened and separated to study their role in maintaining the eco-balance of the oral cavity.

\section{Acknowledgements}

This work was supported by the National Natural Science Foundation of China (grant nos. 91639106, 81270202 and 91339113 to H-B Xin, 31560264 and 81503364 to Tingtao Chen and 21461015 to Xiaolei Wang); the National Basic Research Program of China (grant no. 2013CB531103 to Hongbo Xin); the Open Foundation Of Hubei Key Laboratory Of Lipid Chemistry And Nutrition (grant no. 201602 to Tingtao Chen); the Science Foundation of Jiangxi Provincial Department of Education (grant nos. KJLD14010 and 20153BCB23035 to Xiaolei Wang and 20151BBG70239 to Yan Shi); and, the major program of Natural Science Foundation of Jiangxi Province (grant no. 20161ACB21002 to Xiaolei Wang).

\section{References}

1. Xie G, Chain PS, Lo CC, Liu KL, Gans J, Merritt J and Qi F: Community and gene composition of a human dental plaque microbiota obtained by metagenomic sequencing. Mol Oral Microbiol 25: 391-405, 2010.

2. Palmer RJ Jr: Composition and development of oral bacterial communities. Periodontol 2000 64: 20-39, 2014.

3. Marsh PD: Microbial ecology of dental plaque and its significance in health and disease. Adv Dent Res 8: 263-271, 1994.

4. Seymour GJ, Ford PJ, Cullinan MP, Leishman S and Yamazaki K: Relationship between periodontal infections and systemic disease. Clin Microbiol Infec 13 (Suppl 4): S3-S10, 2007. 
5. Sturgeon A, Stull JW, Costa MC and Weese JS: Metagenomic analysis of the canine oral cavity as revealed by high-throughput pyrosequencing of the 16S rRNA gene. Vet Microbiol 162: 891-898, 2013.

6. Sibley CD, Peirano G and Church DL: Molecular methods for pathogen and microbial community detection and characterization: Current and potential application in diagnostic microbiology. Infect Genet Evol 12: 505-521, 2012.

7. Botelho MP, Maciel SM, Cerci Neto A, Dezan CC, Fernandes KB and de Andrade FB: Cariogenic microorganisms and oral conditions in asthmatic children. Caries Res 45: 386-392, 2011.

8. Allaker RP and Ian Douglas CW: Non-conventional therapeutics for oral infections. Virulence 6: 196-207, 2015.

9. McGuckin M, Goldman R, Bolton L and Salcido R: The clinical relevance of microbiology in acute and chronic wounds. Adv Skin Wound Care 16: 12-23; quiz 24-25, 2003.

10. Thomson PD: Immunology, microbiology, and the recalcitrant wound. Ostomy Wound Manage 46 (1A Suppl): 77S-82S; quiz 83S-84S, 2000.

11. Whitley R: The new age of molecular diagnostics for microbial agents. N Engl J Med 358: 988-989, 2008.

12. Davies CE, Hill KE, Wilson MJ, Stephens P, Hill CM, Harding KG and Thomas DW: Use of $16 \mathrm{~S}$ ribosomal DNA PCR and denaturing gradient gel electrophoresis for analysis of the microfloras of healing and nonhealing chronic venous leg ulcers. J Clin Microbiol 42: 3549-3557, 2004.

13. Kobayashi N, Bauer TW, Tuohy MJ, Lieberman IH, Krebs V, Togawa D, Fujishiro T and Procop GW: The comparison of pyrosequencing molecular Gram stain, culture, and conventional Gram stain for diagnosing orthopaedic infections. J Orthop Res 24: 1641-1649, 2006.

14. Zhang C, Zhang M, Pang X, Zhao Y, Wang L and Zhao L: Structural resilience of the gut microbiota in adult mice under high-fat dietary perturbations. ISME J 6: 1848-1857, 2012.

15. Wang T, Cai G, Qiu Y, Fei N, Zhang M, Pang X, Jia W, Cai S and Zhao L: Structural segregation of gut microbiota between colorectal cancer patients and healthy volunteers. ISME J 6 : 320-329, 2012.

16. Selwitz RH, Ismail AI and Pitts NB: Dental caries. Lancet 369 51-59, 2007.

17. Näse L, Hatakka K, Savilahti E, Saxelin M, Pönkä A, Poussa T, Korpela R and Meurman JH: Effect of long-term consumption of a probiotic bacterium, Lactobacillus rhamnosus GG, in milk on dental caries and caries risk in children. Caries Res 35: 412-420, 2001.

18. Goepfert AR, Jeffcoat MK, Andrews WW, Faye-Petersen O, Cliver SP, Goldenberg RL and Hauth JC: Periodontal disease and upper genital tract inflammation in early spontaneous preterm birth. Obstet Gynecol 104: 777-783, 2004
19. Liu B, Faller LL, Klitgord N, Mazumdar V, Ghodsi M, Sommer DD, Gibbons TR, Treangen TJ, Chang YC, Li S, et al: Deep sequencing of the oral microbiome reveals signatures of periodontal disease. PLoS One 7: e37919, 2012.

20. Faveri M, Mayer MP, Feres M, de Figueiredo LC, Dewhirst FE and Paster BJ: Microbiological diversity of generalized aggressive periodontitis by $16 \mathrm{~S}$ rRNA clonal analysis. Oral Microbiol Immunol 23: 112-118, 2008

21. Hirotomi T, Yoshihara A, Ogawa H, Ito K, Igarashi A and Miyazaki H: A preliminary study on the relationship between stimulated saliva and periodontal conditions in community-dwelling elderly people. J Dent 34: 692-698, 2006.

22. Yu X, Wu X, Qiu L, Wang D, Gan M, Chen X, Wei H and $\mathrm{Xu} F$ : Analysis of the intestinal microbial community structure of healthy and long-living elderly residents in Gaotian Village of Liuyang City. Appl Microbiol Biotechnol 99: 9085-9095, 2015.

23. Xu J, Lian F, Zhao L, Zhao Y, Chen X, Zhang X, Guo Y, Zhang C, Zhou Q, Xue Z, et al: Structural modulation of gut microbiota during alleviation of type 2 diabetes with a Chinese herbal formula. ISME J 9: 552-562, 2015.

24. Cheng M, Qian L, Shen G, Bian G, Xu T, Xu W, Shen G and $\mathrm{Hu}$ S: Microbiota modulate tumoral immune surveillance in lung through a $\gamma \delta T 17$ immune cell-dependent mechanism. Cancer Res 74: 4030-4041, 2014.

25. Alcaraz LD, Belda-Ferre $\mathrm{P}$, Cabrera-Rubio R, Romero $\mathrm{H}$, Simón-Soro A, Pignatelli M and Mira A: Identifying a healthy oral microbiome through metagenomics. Clin Microbiol Infect 18 (Suppl 4): S54-S57, 2012.

26. Loesche WJ: Role of Streptococcus-mutans in human dental decay. Microbiol Rev 50: 353-380, 1986.

27. Corby PM, Lyons-Weiler J, Bretz WA, Hart TC, Aas JA, Boumenna T, Goss J, Corby AL, Junior HM, Weyant RJ and Paster BJ: Microbial risk indicators of early childhood caries. J Clin Microbiol 43: 5753-5759, 2005.

28. Costerton JW, Stewart PS and Greenberg EP: Bacterial biofilms: A common cause of persistent infections. Science 284: 1318-1322, 1999.

29. Ley RE, Turnbaugh PJ, Klein S and Gordon JI: Microbial ecology: Human gut microbes associated with obesity. Nature 444: 1022-1023, 2006.

30. Stackebrandt E, Murray R and Trüper H: Proteobacteria classis nov., a name for the phylogenetic taxon that includes the "purple bacteria and their relatives'. Int J Syst Bacteriol 38: 321-325, 1988.

31. Frank DN and Pace NR: Molecular-phylogenetic analyses of human gastrointestinal microbiota. Curr Opin Gastroenterol 17: $52-57,2001$. 